\title{
Observation of the symmetry breaking of the Fermi Pasta Ulam recurrence in optical fibers
}

\author{
Arnaud Mussot ${ }^{1}$, Corentin Naveau ${ }^{1}$, Pascal Szriftgiser $^{1}$, Matteo Conforti ${ }^{1}$, Alexandre Kudlinski ${ }^{1}$, \\ François Copie $^{1}$, and Stefano Trillo ${ }^{2}$ \\ 1. Univ. Lille, CNRS, UMR 8523-PhLAM - Physique des Lasers Atomes et Molécules, F-59000 Lille, France \\ 2. Department of Engineering, University of Ferrara, Via Saragat 1, 44122 Ferrara, Italy
}

\begin{abstract}
We provide the first longitudinal characterization in phase and amplitude of the Fermi Pasta Ulam recurrence in optical fibers by means of a heterodyne optical time domain reflectometer. This original setup allows to report the symmetry breaking of the process due to an initial condition change. (c) 2018 The Author(s)

OCIS codes: (190.4370) Nonlinear optics, fibers; 190.4380 Nonlinear optics, four-wave mixing; 190.4410 Nonlinear optics, parametric processes
\end{abstract}

Fermi Pasta Ulam (FPU) recurrence process is a universal phenomenon that describes the natural return cycle of a nonlinear system into its initial state after experiencing a complex nonlinear coupling dynamics. In optical fibers it has been observed with reference to the nonlinear stage of modulation instability (MI) [1,2], which also plays a key role in supercontinuum and rogue wave formation. However, the nonlinear MI is characterized by a broken symmetry [3,4], which entails that, in a suitable phase-space, two qualitatively different types of evolutions can be followed by evolving from the modulated pump to a comb of sideband pairs and back to the pump. To date, the observation of such a broken symmetry has been elusive because of the intrinsic challenge of measuring the longitudinal evolution of the relative phase of the comb lines.
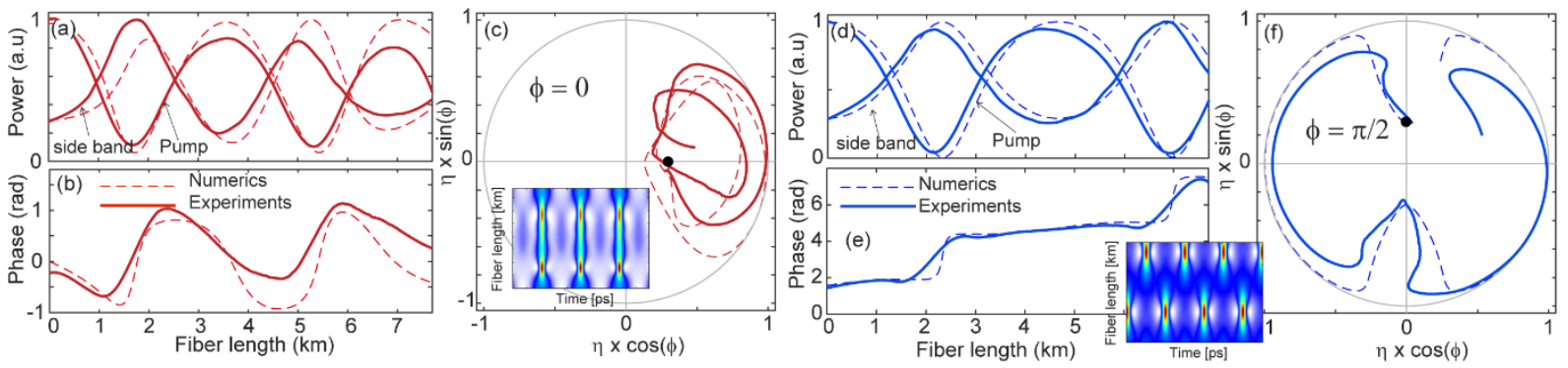

Fig. 1 Evolution with fiber length (a) of the normalized power of the pump and of the sideband, (b) of the pump-signal relative phase ( $\Phi)$ ). (c) Phase portrait of the sideband corresponding to (a) and (b). (a) to (c) AM case ( $\Phi=0$, red lines) and (d) to (f) FM case ( $\Phi=\pi / 2$, blue lines). Dashed lines represent numerical simulations and solid lines experimental results. Insets: temporal evolution of the intensity from simulations. Parameters: $\beta_{2}=-19 \times 10^{-27} \mathrm{~s}^{2} / \mathrm{m}, \gamma=1.3 / \mathrm{W} / \mathrm{km}$, pump power $450 \mathrm{~mW}$, sideband power $63.6 \mathrm{~mW}$, sideband detuning $35 \mathrm{GHz}, \lambda_{\mathrm{P}}=1555 \mathrm{~nm}$, fiber length $7.7 \mathrm{~km}$. Linear losses are compensated by means of distributed Raman amplification. Powers are normalized to maximum values. $\eta(\mathrm{z})$ is the normalized sideband power, $\phi(\mathrm{z})$ is the relative phase between one of the sideband and the pump.

In this work, we report the first complete experimental observation of the broken symmetry of FPU recurrence associated with MI in optical fibers by means of a heterodyne optical time domain reflectometer (HOTDR). The HOTDR allows for recording the two qualitatively different phase portraits of FPU recurrence, contrasted in Fig. $1(a, b, c)$ and (d,e,f), respectively, and accessible by changing the input modulation phase. Fig. 1(a) displays the longitudinal evolution of the normalized pump and sideband powers, and Fig. 1(b) their relative phase, when a pure amplitude modulation is injected at the fiber input (in-phase input, $\phi(0)=0)$. First, two whole cycles of recurrence have been experimentally observed which is unprecedented in optical fiber systems. Second, the projection onto the phase-plane in Fig. 1(c) clearly shows that the relative phase remains confined in the right semi-plane $(-\pi / 2<\phi<\pi / 2)$, meaning that maximum compression points are in phase in the time domain (see inset calculated from numerical simulations). Conversely, by changing the input phase to $\phi(0)=\pi / 2$ (pure frequency modulation) a completely different scenario occurs (Fig. 1(d,e,f)). In this case, the two successive recurrences alternate in the right and left semi-planes, corresponding to a $\pi$ phase shift between maximum recompression points in the time domain (see inset in (f) from numerics). The two phase portraits in Fig. 1(c) and (f) clearly correspond to the equivalent motion of a particle exploring either one of the wells or both wells of a double-well potential, which is the accessible signature of the broken symmetry associated with the nonlinear MI ruled by the nonlinear Schrodinger equation. The HOTDR reveals these two evolutions in the same system, simply by changing the input modulation phase. This is a key feature related to the conservative nature of the dynamics which is preserved in our system due to Raman-compensation of the fiber losses. In this sense, our results differ from the 
observation of shifted recurrence of MI observed recently in a deep water tank, which is induced by the unavoidable losses [5].

In summary, our original experimental technique permitted to reveal the symmetry breaking of the Fermi Pasta Ulam recurrence process via an initial condition change [6]. This opens the way to further experimental investigations ranging from rogue-wave/supercontinuum formation to complex cavity dynamic characterisation where modulation instability plays a fundamental role.

\section{References}

1. G. Van Simaeys, P. Emplit, and M. Haelterman, "Experimental Demonstration of the Fermi-Pasta-Ulam Recurrence in a Modulationally Unstable Optical Wave," Phys. Rev. Lett. 87, 033902 (2001).

2. N. N. Akhmediev, "Nonlinear physics: Déjà vu in optics," Nature 413, 267-268 (2001).

3. N. N. Akhmediev and V. I. Korneev, "Modulation instability and periodic solutions of the nonlinear Schrödinger equation," Teor Mat Fiz 69, 189-194 (1986).

4. S. Trillo and S. Wabnitz, "Dynamics of the nonlinear modulational instability in optical fibers," Opt. Lett. 16, 986-988 (1991)

5. O. Kimmoun, H. C. Hsu, H. Branger, M. S. Li, Y. Y. Chen, C. Kharif, M. Onorato, E. J. R. Kelleher, B. Kibler, N. Akhmediev, and A. Chabchoub, "Modulation Instability and Phase-Shifted Fermi-Pasta-Ulam Recurrence," Sci. Rep. 6, 28516 (2016).

6. A. Mussot, C. Naveau, P. Szriftgiser, M. Conforti, A. Kudlinski, F. Copie, and S. Trillo, "Multi-wave mixing combs reveal the broken symmetry of Fermi-Pasta- Ulam recurrence", to appear in Nature photonics (2018). 